\title{
The relation between tender points and fibromyalgia symptom variables: evidence that fibromyalgia is not a discrete disorder in the clinic
}

Frederick Wolfe

\begin{abstract}
Objective-To investigate the relation between measures of pain threshold and symptoms of distress to determine if fibromyalgia is a discrete construct/ disorder in the clinic.
\end{abstract}

Methods-627 patients seen at an outpatient rheumatology centre from 1993 to 1996 underwent tender point and dolorimetry examinations. All completed the assessment scales for fatigue, sleep disturbance, anxiety, depression, global severity, pain, functional disability, and a composite measure of distress constructed from scores of sleep disturbance, fatigue, anxiety, depression, and global severity-the rheumatology distress index (RDI).

Results-In regression analyses, the RDI was linearly related to the count of tender points $\left(r^{2}=0.30\right)$. Lesser associations were found between the RDI and dolorimetry measurements $\left(r^{2}=0.08\right)$. The RDI was more strongly correlated with the two measures of pain threshold than any of the individual fibromyalgia symptom variables. In partial correlation analyses, all of the information relating to symptom variables was contained in the tender point count, and dolorimetry was not independently related to symptoms.

Conclusion-Tender points are linearly related to fibromyalgia variables and distress, and there is no discrete enhancement or perturbation of fibromyalgia or distress variables associated with very high levels of tender points. Although fibromyalgia is a recognisable clinical entity, there seems to be no rationale for treating fibromyalgia as a discrete disorder, and it would seem appropriate to consider the entire range of tenderness and distress in clinic patients as well as in research studies. The tender point count functions as a 'sedimentation rate' for distress, and is a better measure than the dolorimetry score.

(Ann Rheum Dis 1997;56:268-271)
Fibromyalgia represents the intersection of a considerably abnormal and reduced pain threshold with a series of clinical distress variables, including pain, fatigue, sleep disturbance, anxiety, and depression, among others. In the clinic, it is best diagnosed by counting the number of tender points a patient has. In the presence of 11 or more tender points and widespread pain, fibromyalgia is diagnosed (classified) according to American College of Rheumatology (ACR) Criteria. ${ }^{1}$

The ability to diagnose fibromyalgia with commonly agreed upon criteria has stimulated research into basic and clinic aspects of the syndrome. In general, research has used 'normals' or patients with other rheumatic diseases as control subjects. This comparison, of fibromyalgia with such control subjects, implies that fibromyalgia is a discrete entity. However, epidemiological studies suggest, instead, that fibromyalgia may be merely the end of a continuum of distress. ${ }^{23}$ Epidemiologically defined disease may be different from clinically defined disease, and the issue of whether fibromyalgia is a relatively discrete clinical entity has not been investigated in the clinic. This is an important question, because if fibromyalgia does represent a clinical as well as an epidemiological continuum, then we may be failing to identify many patients in the clinic with syndromes similar to fibromyalgia, though with fewer symptoms or tender points. In addition, in characterising patients as having or not having fibromyalgia we may be missing, in those with not enough tender points, important symptoms of distress. Finally, we may be concentrating basic and clinical research inappropriately into a constricted area of a pain-distress continuum.

We investigated the question of whether fibromyalgia is a relatively discrete clinical entity in 627 clinic patients by obtaining measures of fibromyalgia symptoms as well as physical measures of tender point counts and dolorimetry scores.

\section{Methods}

SUBJECTS

Subjects in this study were 627 patients seen at an outpatient rheumatology centre (Wichita Arthritis Center) during a period of three and 
half years from 22 February 1993 to 23 August 1996. Patients consisted of two groups, 374 patients seen before 1 August 1993 as part of a project to examine serial patients returning for follow up visits and 253 patients seen after that date in whom the examinations were made for the purpose of clinical diagnosis.

PHYSICAL EXAMINATION DATA

All patients underwent a count of tender points using the 18 sites specified in the American College of Rheumatology 1990 Classification criteria for fibromyalgia. ${ }^{1}$ Tender point data are reported as a count of positive tender point sites. In addition, each patient had a dolorimetry examination performed at the trapezii, knees, lateral epicondyle, and second rib using the Fischer Dolorimeter (Pain Diagnostics and Thermography, Great Neck, NY) with a one centimetre in diameter rubber tip. A dolorimeter is a pressure algometer. To use it, the examiner places the rubber tip on the examination site and gradually increases the pressure at a rate of approximately $1 \mathrm{~kg} / \mathrm{cm}^{2}$ per second. The patient is asked to report the moment when the sensation at the examination site changes from that of pressure to that of pain. At that point, the force is recorded in $\mathrm{kg}$. The reported dolorimetry score is the mean of the sites examined. Dolorimetry values are thought to be a measure of pain threshold. Dolorimetry scores represent a continuum in the population, with median values for women of $4.25 \mathrm{~kg} / \mathrm{cm}^{2}$ and $6.0 \mathrm{~kg} / \mathrm{cm}^{2}$ for men being reported using the same Fisher dolorimeter and methodology. ${ }^{4}$ Among persons with fibromyalgia in a population survey, mean dolorimetry scores were approximately $2.7 \mathrm{~kg} / \mathrm{cm}^{2}{ }^{3}$

QUESTIONNAIRE DATA

The Clinical Health Assessment Questionnaire (CLINHAQ) was used for each patient. ${ }^{5}$ This instrument contains self reports for the Health Assessment Questionnaire (HAQ) disability index, ${ }^{67}$ arthritis impact measurement scales (AIMS) anxiety and depression index, ${ }^{8}$ visual analogue scale (VAS) pain, VAS global severity, VAS gastrointestinal symptoms, VAS sleep problems, VAS fatigue, satisfaction with health



Figure 1 Graph of rheumatology distress index versus tender point count scores. Lines are predicted lowess (locally weighted regression) lines and 95\% confidence intervals. The $\mathrm{r}^{2}$ values from linear regressions are 0.30 and 0.08 , respectively. and patient estimate of health status. In 1996, the helplessness subscale of the rheumatology attitudes index (RAI) was added to the CLINHAQ. ${ }^{9}$ The variables contained in this instrument consider factors that are thought to be of major importance in fibromyalgia. ${ }^{1011}$

The specific fatigue assessment used a $15 \mathrm{~cm}$ double anchored VAS labelled on one end, 'Fatigue is no problem' and on the other end, 'Fatigue is a major problem.' The question read 'How much of a problem has fatigue or tiredness been for you in the past week?' The range of the scale is $0-3$. The specific questions and anchors for the other $15 \mathrm{~cm}$ VAS scales were pain: 'How much pain have you had because of your illness in the past week?' (no pain, severe pain); global severity: 'Consider all of the ways that your illness affects you, rate how you are doing by placing a mark on the line' (very well, very severe); sleep problems: 'How much problem has sleep (ie, resting at night) been for you in the past week?' (sleep is no problem, sleep is a major problem). Except for global severity, which is scored 0-100, all other VAS scales are scored 0-3.

The rheumatology distress index (RDI) is computed from questionnaire variables described above. It is an approximate linear combination of questionnaire variables that most accurately identify (a) distressed patients and (b) those with fibromyalgia in comparison to a large series of other questionnaire clinical, demographic, and psychological variables. ${ }^{112}$ It is computed through the following formula: rheumatology distress index $=(($ anxiety/9.9 $)+$ $($ depression/9.9) $+($ global severity/100) + (sleep disturbance/3) $+($ fatigue/3) $) \times 20$. The divisors for each scale convert the variable to a 0-1 range. For example, the AIMS depression and anxiety scales have a range of 0-9.9. Dividing by 9.9 converts the scales to $0-1$. The five variable scores are then added, producing a scale with a range of $0-5$. After multiplication by 20 the range of scores is from 0 (no abnormality on any subscale) through 100 (maximum abnormality on all subscales). In this study, the RDI was approximately normally distributed with a mean of 46.5 and a standard deviation of 20.7. To test the appropriateness of the RDI index, a new variable that represented the first principal component of the RDI variables (anxiety, depression, global severity, sleep disturbance, and fatigue) was created and then compared with the RDI result. The correlation between RDI and tender point count was 0.55 , and the correlation between the new principal component variable and tender point count was 0.55 . Therefore the index is an appropriate composite measure of the five variables.

\section{STATISTICAL ANALYSES}

Data were analysed using Intercooled Stata version 5.0 for Windows. ${ }^{12}$ Pearson correlations coefficients were used. To test the equality of dependent correlations we used the Goldstein implementation of the Fischer $z$ transformation. ${ }^{13}$ Data were analysed by least squares linear regression and by lowess (locally weighted regression) regression using a narrow 




Figure 2 Graph of rheumatology distress index versus dolorimetry scores. Lines are predicted lowess (locally weighted regression) lines and 95\% confidence intervals. The $\mathrm{r}^{2}$ values from linear regressions are 0.30 and 0.08 , respectively.

bandwidth of $0.4 .^{14}$ Lowess regression is very sensitive to local changes, and would be expected to identify, by alteration of the prediction line, specific associations between RDI and pain threshold at tender point counts of 11 or more. Statistical significance was set at 0.05. All tests were two sided.

\section{Results}

Of the 627 patients, there were 267 patients with a primary diagnosis of fibromyalgia, 156 with rheumatoid arthritis (RA), and 182 with osteoarthritis (OA). Of the RA patients, $22.4 \%$ had 11 or more tender points; $24.73 \%$ of OA patients had 11 or more tender points, and $89.9 \%$ of those diagnosed with fibromyalgia (including $95.8 \%$ of fibromyalgia patients seen for the first time in the clinic) satisfied the ACR tender point criterion. ${ }^{1}$ Patients not having RA, OA or fibromyalgia $(n=22)$ had other disorders such low back pain, tendonitis, arthralgias, and miscellaneous inflammatory disorders.

The RDI was directly related to the count of tender points (fig 1). The $r^{2}$ of the regression of RDI on tender point count was 0.30 , and the estimated $\beta$ coefficient was 1.89 , SEM 0.11 , $\mathrm{p}<0.001$. Thus, on the average, an increase of one tender point is associated with a two unit increase in RDI, and a 10 unit increase in tender points with a 20 unit increase in RDI. By contrast, the $r^{2}$ for the regression of RDI on dolorimetry score was 0.08 (fig 2). Therefore dolorimetry is a poor predictor of distress. The

Table 1 Pearson correlation of tender point count with clinical severity and distress variables

\begin{tabular}{lcc}
\hline & Tender point count & Dolorimetry score \\
\hline Tender point count & 1.000 & \\
Rheumatology distress index & 0.550 & $-0.288^{\star}$ \\
Dolorimetry score & -0.522 & $1.000^{\star}$ \\
Fatigue scale & 0.479 & $-0.231^{\star}$ \\
Anxiety index & 0.458 & $-0.236^{\star}$ \\
Sleep disturbance scale & 0.411 & $-0.234^{\star}$ \\
Pain scale & 0.404 & $-0.253^{\star}$ \\
Global severity & 0.399 & $-0.231^{\star}$ \\
Depression index & 0.396 & $-0.182^{\star}$ \\
HAQ disability index & 0.309 & -0.301 \\
\hline All correlation coefficients are significant at $\mathrm{p}<0.001 .^{\star}$ Indicates that the correlation \\
coefficients for tender point count and dolorimetry score with the variable in the first column are \\
different at the 0.05 level.
\end{tabular}

tender point count is a better predictor, explaining $30 \%$ of $\mathrm{RDI}$ variance.

To further understand the relation of the tender point count and dolorimetry variables we determined their correlation with other clinical severity and distress variables. As expected, the correlation between the tender point count and RDI was the strongest. Importantly, for all variables except HAQ disability index, tender point count had a significantly stronger correlation than did dolorimetry score.

Next, partial correlation analysis was used to determine the strength of the association of either tender point count or dolorimetry with RDI, after controlling for dolorimetry and tender point count, respectively. Controlling for dolorimetry, the association between RDI and tender point count was $r=0.489, \mathrm{p}<0.001$. Controlling for tender point count, the association between RDI and dolorimetry was $r=-0.001, \mathrm{p}=0.976$ (table 1$)$.

\section{Discussion}

As expected, we found associations between fibromyalgia variables (fatigue, sleep, anxiety, depression, global severity, pain) and the tender point count, and we found the strongest association with the composite distress variable, RDI. As shown in figure 1, a linear relation between tender points and RDI exists throughout the entire range of values. In addition, there are high values for RDI in those patients having 11 or more tender points, as would be expected among fibromyalgia patients. We believe these data show that there is a parallel continuum of distress and tender points, and that there is no discrete enhancement or perturbation of fibromyalgia or distress variables associated with very high levels of tender points. That is, the relation between clinical symptoms and tender points is linear, not quadratic. In addition, our findings - that intermediate levels of tender points may be associated with clinical distress, support the data of Middleton et al in a population of systemic lupus erythematosus patients. ${ }^{15}$

We also found that pain threshold as measured by dolorimetry scores was generally poorly correlated with clinical symptoms. This finding extends a similar observation we made in an epidemiological population survey. ${ }^{4}$ Dolorimetry can be conceived of as a pure measure of pain threshold, while the tender point count seems to have increased psychological or distress associated symptom content. In this study, partial correlation analysis showed that dolorimetry was not independently correlated with RDI.

These findings suggest, then, that fibromyalgia tenderness and symptoms are part of a continuum. Physicians will diagnose fibromyalgia when the symptoms and tenderness reach the physician's threshold, and it seems clearly appropriate and useful to do this in the clinic, just as it is appropriate and useful to use the ACR classification criteria. ${ }^{1}$ But in a broader sense, there is no discrete point where fibromyalgia does or does not exist; and it is important 
to recognise the importance of distress symptoms whether or not the patient reaches the fibromyalgia diagnostic threshold.

The implications of our data may be important to rheumatologists and others in the medico-legal arena where fibromyalgia is often assumed to be a discrete disease and trauma may be though to be causally related. Our data would suggest that fibromyalgia is not a discrete disease, and that it is just as rationale to associate (or not associate) trauma with five tender points or 10 tender points or the requisite 11 or more tender points. Similarly, for basic research, there seems to be no rationale for treating fibromyalgia as a discrete disorder, and it would seem more appropriate in such studies to examine the entire range of tenderness and distress.

Supported in part by grants (AM21393) from the National Institutes of Health to the Arthritis, Rheumatism and Aging, Medical Information Center (ARAMIS).

1 Wolfe F, Smythe HA, Yunus MB, et al. The American College of Rheumatology 1990 Criteria for the Classification of Fibromyalgia: Report of the Multicenter Criteria Committee. Arthritis Rheum 1990;33:160-72.

2 Croft P, Schollum J, Silman A. Population study of tender point counts and pain as evidence of fibromyalgia. BM 1994;309:696-9

3 Wolfe F, Ross K, Anderson J, Russell IJ, Hebert L. The prevalence and characteristics of fibromyalgia in the general population. Arthritis Rheum 1995;38:19-28.
4 Wolfe F, Ross K, Anderson J, Russell IJ. Aspects of fibromyalgia in the general population: Sex, pain threshold, and fibromyalgia symptoms. J Rheumatol 1995;22:151-6.

5 Wolfe F. Data collection and utilization: a methodology for clinical practice and clinical research. In: Wolfe F, Pincus $\mathrm{T}$, eds.Rheumatoid arthritis: pathogenesis, assessment, outcome, and treatment. New York: Marcel Dekker, 1994:463come, an 514 .

6 Fries JF, Spitz PW, Kraines RG, Holman HR. Measurement of patient outcome in arthritis. Arthritis Rheum 1980; 23:137-45.

7 Wolfe F, Kleinheksel SM, Cathey MA, Hawley DJ, Spitz PW, Fries JF. The clinical value of the Stanford Health Assessment Questionnaire Functional Disability Index in patients with rheumatoid arthritis. J Rheumatol 1988; 15:1480-8.

8 Hawley DJ, Wolfe F. Depression is not more common in rheumatoid arthritis: a 10 year longitudinal study of 6,608 rheumatic disease patients. J Rheumatol 1993;20:202531.

9 deVellis RF, Callahan LF. A brief measure of helplessness in rheumatic disease - the helplessness subscale of the rheumatology attitudes index. J Rheumatol 1993;20:866-9.

10 Burckhardt CS, Clark SR, Bennett RM. The fibromyalgia impact questionnaire: development and validation. J Rheumatol 1991;18:728-33.

11 Simms RW, Felson DT, Goldenberg DL. Development of preliminary criteria for response to treatment in fibromyalgia syndrome. J Rheumatol 1991;18:1558-63.

12 Stata Corporation. Stata Statistical Software: Release 5.0. College Station, TX: Stata Corporation, 1997.

13 Goldstein R. Testing dependent correlation coefficients. Stata Technical Bulletin. 1996;32.

14 Cleveland WS. Robust locally-weighted regression and smoothing scatterplots. J Am Statist Assoc 1979;74:82936.

15 Middleton GD, Mcfarlin JE, Lipsky PE. The prevalence and clinical impact of fibromyalgia in systemic lupus erythematosus. Arthritis Rheum 1994;37:1181-8. 